\title{
Hemangioblastoma in the Setting of Von Hippel Lindau Disease: A Case Report
}

\author{
Raajini Devi $\mathrm{K}^{1}$, Aida Zairani $\mathrm{MI}^{1}$, Hazlita $\mathrm{MI}^{1}$, Jemaima $\mathrm{CH}^{1}$, Farizal F${ }^{2}$, Safinaz $\mathrm{MK}^{1}(\bowtie)$ \\ ${ }^{1}$ Department of Ophthalmology, ${ }^{2}$ Department of Neurosurgery, Faculty of Medicine, Universiti \\ Kebangsaan Malaysia Medical Centre, Jalan Yaacob Latif, Bandar Tun Razak, 56000 Cheras, Kuala \\ Lumpur, Malaysia.
}

\begin{abstract}
A 21-year-old Chinese gentleman with no known medical illness, presented with a history of right painless blurring of vision with central scotoma of two weeks duration. He also had a history of multiple episodes of seizures prior to presentation. Visual acuity was $1 / 60$ with unremarkable anterior segment findings and no relative afferent pupillary defect. Fundus examination of the right eye revealed dilated and tortuous retinal veins with multiple retinal capillary hemangiomas and sub retinal hard exudates at the macula with edema. A diagnosis of Von Hippel Lindau disease was made when a posterior fossa mass suggestive of hemangioblastoma with obstructive hydrocephalus was seen on computed tomography of the brain. Craniotomy with nodule excision was performed. The retinal capillary hemangiomas were treated with the combination of laser photocoagulation and intravitreal Ranibizumab injections. Visual acuity subsequently improved to 6/36.
\end{abstract}

Keywords: craniotomy, hemangioblastoma, hemangioma, photocoagulation, scotoma, Von Hippel Lindau disease

\section{Correspondence:}

Safinaz Mohd Khialdin, Department of Ophthalmology, Faculty of Medicine, Universiti Kebangsaan Malaysia Medical Centre, Jalan Yaacob Latif, Bandar Tun Razak, 56000 Cheras, Kuala Lumpur, Malaysia. Tel: +603-9145 5981 Fax: +60391456733 Email:drsafinaz_1978@yahoo.com.my

\section{Introduction}

Von Hippel Lindau (VHL) disease is a highly penetrant, heritable, autosomal dominant multisystem cancer syndrome. The germ line mutation of the Von Hippel Lindau tumour suppressor gene is located on the short arm of chromosome 3. Affected individuals may present with central nervous system (CNS) and retinal haemangioblastomas, renal clear cell carcinoma, phaeochromocytoma and also endolymphatic sac tumours (1). We report a case with retinal haemangioblastoma with history of presumed seizure episodes, alarming the possibility of VHL disease.

\section{Case Report}

A 21-year-old Chinese gentleman complained of painless blurring of vision in the right eye associated with central scotoma for two weeks prior to presentation. He reported history of multiple episodes of seizures. He never sought any treatment for these presumed-seizure episodes, and thus was never properly investigated.

At presentation, visual acuity in the right eye was 1/60 and 6/6 in the left eye. Anterior segments findings in the right eye were normal with no relative afferent pupillary defect. Retinal capillary haemangiomas (RCHs) 


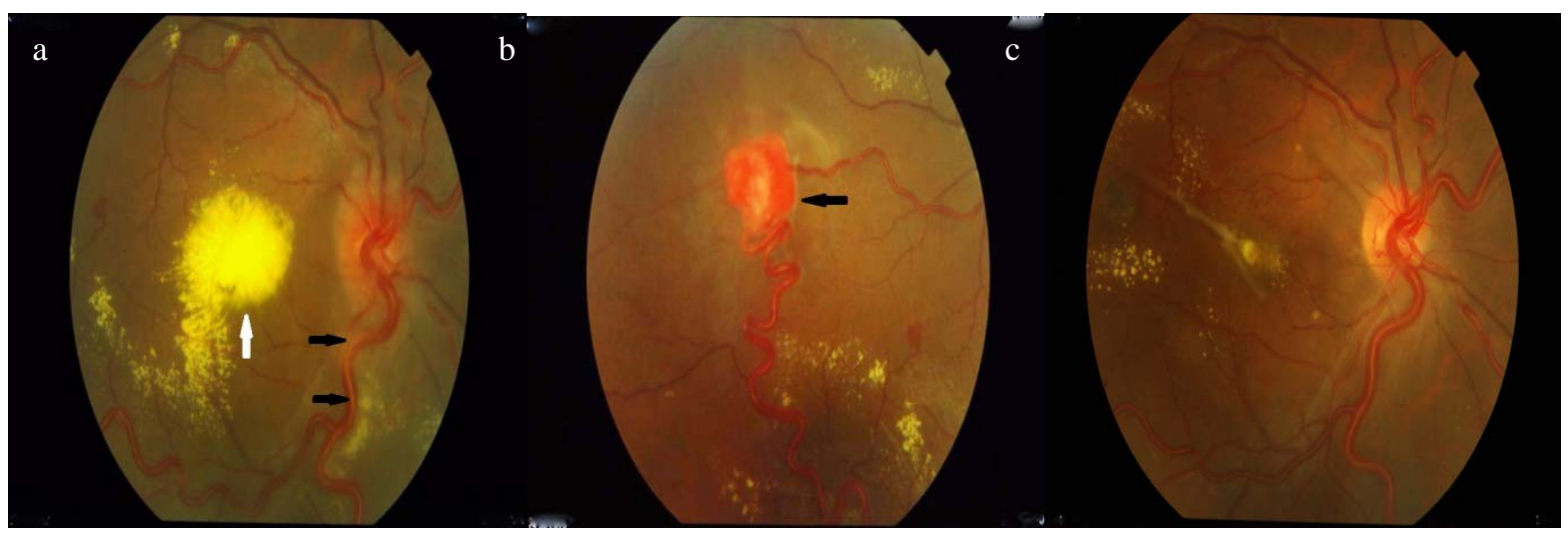

Figure 1: (a) Fundus photographs showing dilated, tortuous retinal veins (black arrow), large subretinal hard exudate at macula with edema (white arrow), (b) RHC on presentation (black arrow) and (c) post- laser and intravitreal Ranibizumab injections.

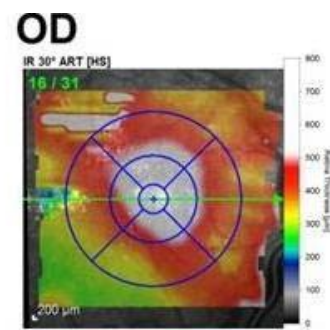

OCT 20 (8. $8.5 \mathrm{~mm})$ ART (ต) Q: 37 [HS]

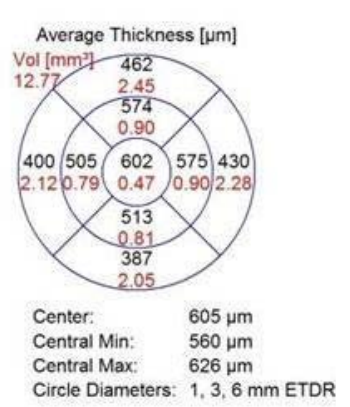

Circle Diameters: $1,3,6 \mathrm{~mm}$ ETDR

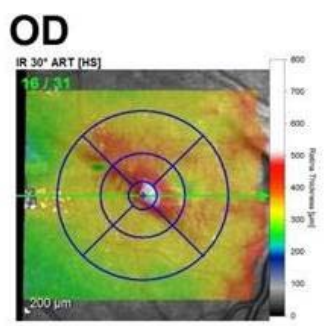

OCT $28.8 .5 \mathrm{~mm})$ ART (11) Q: 28 [Hs

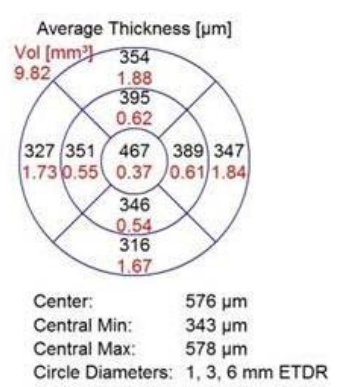

Circle Diameters: $1,3,6 \mathrm{~mm}$ ETDR

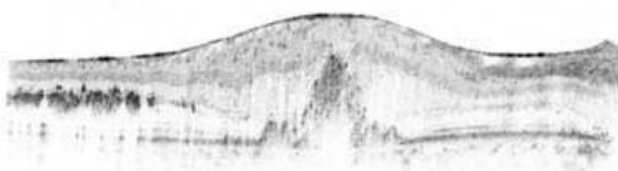

a

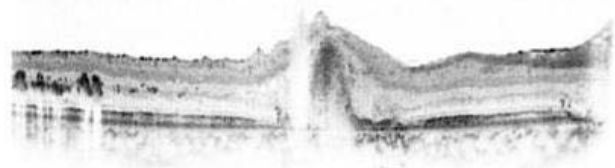

b

Figure 2: (a) OCT macula thickness: pre injection, (b) post injection

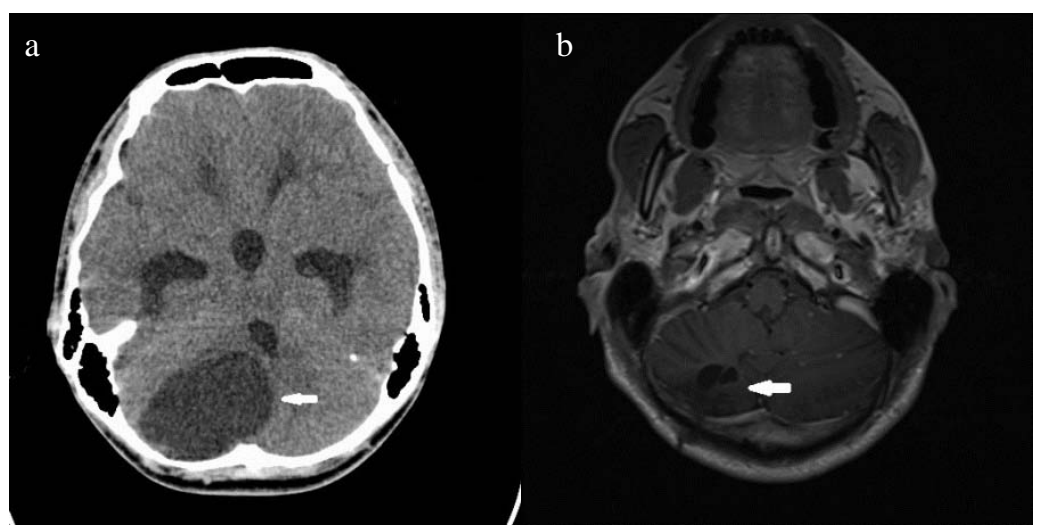

Figure 3: (a) Preoperative cystic posterior fossa tumor and (b) post-op encaphalomalacia with no residual tumor

measuring about 1.5-2 mm associated with dilated and tortuous retinal veins, large multiple subretinal hard exudates at macula with edema were seen in the right eye (Fig. 1). Central macular thickness (CMT) on 
Optical Coherence Tomography (OCT) measured $602 \mu \mathrm{m}$ (Fig. 2a). Left eye was normal. Fundus fluorescein angiogram revealed numerous areas of hyper fluorescence with dilated and tortuous feeder vessels, late leakage and masking over the macula.

Computed tomography (CT) revealed a posterior fossa cystic mass measuring 4.0 × 5.5 x $4.9 \mathrm{~cm}$ with an enhancing mural nodule and retinal lesions suggestive of hemangioblastoma with associated obstructive hydrocephalus (Fig. 3a). A diagnosis of Von Hippel Lindau (VHL) disease was established. Systemic review and ultra-sonogram examination of abdomen were normal.

The RCHs was treated with argon laser photocoagulation. A total of 8 intravitreal Ranibizumab injections were given monthly over a period of 8 months. The RCHs responded to treatment (Fig. 1c). Vision became 6/36 and central scotoma had improved with reduction of CMT to $467 \mu \mathrm{m}$ (Fig. 2b).

Craniotomy for cyst evacuation and nodule excision was performed. Histopathological result had concluded the nodule to be hemangioblastoma World Health Organization (WHO) Grade 1 with no evidence of neoplastic cells. Postoperative MRI showed evidence of residual disease with cystic spaces in the posterior fossa measuring $2.1 \times 3.0 \times 1.5 \mathrm{~cm}$ and intramedullary cervical spinal cord haemangioblastoma measuring 1.5 x $2.5 \mathrm{~cm}$. Repeat craniotomy with complete tumour evacuation was performed (Fig. 3b). 5 cycles of radiotherapy was given for the spinal cord with regression of tumour to a size of $0.5 \times 1.5 \mathrm{~cm}$.

Repeat MRI 6 months later showed evidence of new cerebellar hemangioblastoma. However, he is being managed conservatively as it has remained unchanged on repeated scans. He has been scheduled for a 6monthly MRI of cerebellum and spine and annual catecholamine and abdominal ultrasonography for monitoring.

\section{Discussion}

The affected gene in VHL produces a tumor suppressor product known as pVHL. A germ line mutation at chromosome 3p25 results in cells lacking pVHL. These abnormal cells are associated with the overproduction of angiogenic products, such as erythropoietin, vascular endothelial growth factor (VEGF) and platelet-derived growth factor (PDGF). These cells also do not degrade hypoxia-inducible factors (HIF) in the presence of oxygen. VHL associated tumors have high HIF gene levels in all tumor cells, making them highly vascular (2).

RCHs have been identified as the earliest and the most frequent manifestation of this disease, as high as $60 \%$ with the mean age of 25 years at presentation. RCHs manifests as a solitary tumour, appearing as round and circumscribed retinal lesion with an orange-red hue at any part of the retina. It can be exophytic, endophytic or sessile in nature with presence of subretinal and intraretinal exudation. Less than 3\% of cases present with vitreous or retinal haemorrhage (3). Juxtapapillary type of RCHs is commonly seen along the the optic disc at the temporal quadrant in 11-15\% of VHL disease. Anterior segment rubeosis may be seen at a later stage of the disease. Neuro-ophthalmic manifestations such as optic nerve compression and chiasmal syndrome are rare (3).

Untreated RCHs can lead to sight threatening complications such as retinal exudation with macula edema, and traction with consequent exudative or tractional retinal detachments, as high as $16 \%$ and $9 \%$ respectively (3). Initial close observation of $\mathrm{RCH}$ is recommended in specified cases of small nasally located lesions (up to $500 \mu \mathrm{m}$ ) without exudation, subretinal fluid or juxtapapillary type of RHCs.

Argon laser photocoagulation is the commonest mode of treatment which can be delivered to the feeder vessels, to the tumour itself or both. Clinical responses are seen in $91-100 \%$ of cases (4). Laser photocoagulation is the treatment of choice in posterior RCH which measures $1.5 \mathrm{~mm}$ or smaller, or even up to $4.5 \mathrm{~mm}$. RCHs in this case which is considered in this group showed good response to laser photocoagulation.

Cryotherapy is preferable to photocoagulation when the RCHs are anterior and more than $3.0 \mathrm{~mm}$ with a significant amount of subretinal fluid. Advanced cases may require pars plana vitrectomy. Juxtapapillary $\mathrm{RCH}$ generally carries a poorer visual prognosis (4).

Photodynamic therapy (PDT) with verteporfin has been advocated with sustained visual outcome for 22 months by Rarey and Friberg (5). RCH greater than $4 \mathrm{~mm}$ may benefit from radiotherapy as they often respond poorly to cryotherapy and laser photocoagulation. External beam, proton beam or plaque radiotherapy has been advocated (6).

Anti-vascular endothelial growth factor (VEGF) such as Ranibizumab and Bevazicumab play a critical role in controlling the disease directly by halting VEGF 
expression in the rapidly growing tumors. Intravitreal Ranibizumab monotherapy is demonstrated to achieve the best clinical effect when the lesions are smallest and minimally exudative as they are associated with a high cell turnover and increased sensitivity to antiangiogenic inhibitory effects (7). It can be advocated as many times until complete resolution of exudative macula edema and regression of RCHs, however caution has to be undertaken in cases of preexisting tractional component.

CNS haemangioblastomas (WHO Grade I) represent $0.9-2.1 \%$ of all intracranial neoplasms and are the second commonest presentation of VHL. They are noted to be identical microscopically as well as histologically to the sporadic type, regardless of their location. VHL associated tumors tend to be multiple, whereas the sporadic types are usually solitary. They stand a risk of having new RCH one per 2.1 years (8).

Commonest VHL associated CNS haemangioblastoma is the craniospinal type, involving cerebellum in majority of cases (44-72\%), as observed in this case. Less frequent sites are in the spinal cord (13-50\%), followed by brainstem (10-20\%) and supratentorial region (3-6\%). These tumors typically appear brightly enhancing on MRI with a clear demarcation from the surrounding tissue. Vascular flow voids in the presence of major feeder vessels are often identified (8).

Surgical intervention is always the treatment of choice for CNS tumors, taking into consideration the postoperative morbidity and residual CNS deficits and the risk of repeated surgeries. Generally subsequent surgeries may be considered if the tumor size post initial resection is of larger than $3 \mathrm{~cm}$ and posterior fossa in location.

Subtotally resected tumors respond well to radiotherapy (RT) in $60 \%-90 \%$ of cases showing local regression. Resected base with a uniformly symmetrical margin of $1 \mathrm{~cm}$ should be included as the clinical target volume (CTV), with a prescription dose within the range 50-60 Gy having per fraction value of 1.8 or $2.0 \mathrm{~Gy}$ (9). High doses in cases of smaller tumor volume is associated with a better response rate radiation however still carries a risk of peritumoral edema and radiation necrosis.

Unresectable spinal cord hemangioblastoma has been shown to produce significant tumor regression when treated with intravenous bevacizumab therapy (10).

\section{Conclusion}

RCHs are commonly the earliest presentation of VHL, as in this scenario. Therefore, when RCHs are confirmed in a patient, further detailed history and a thorough systemic review is vital, in making a precise diagnosis with a multidisciplinary approach. This is important as VHL disease can lead to sight and life threatening complications if left undiagnosed and untreated at the right time.

\section{References}

1. Lonser RR, Glenn GM, Walther M, et al .von Hippel-Lindau disease. Lancet 2003; 361(9374): 2059-67.

2. Davis S, Uwaydat S. Diagnosis and treatment of von Hippel-Lindau syndrome. Curr Opin Ophthalmol 2008; (19): 213-7.

3. Richard S, Gardie B, Couvé S, Gad S. Von Hippel-Lindau: how a rare disease illuminates cancer biology. Semin Cancer Biol 2013; 23(1): 26-37.

4. Singh AD, Nouri M, Shields CL, Shields JA, Smith AF. Retinal capillary hemangioma: a comparison of sporadic cases and cases associated with von Hippel-Lindau disease. Ophthalmology 2001; 108(10): 1907-11.

5. Rarey K, Friberg TR. Indirect laser treatment and anti-VEGF therapy of a retinal angioma, with resolution of a large serous retinal detachment, macular exudates, and macular edema. Semin Ophthalmol 2010; 25(1-2): 21-6.

6. Singh AD, Nouri M, Shields CL, Shields JA, Perez N. Treatment of retinal capillary hemangioma. Ophthalmology 2002; 109(10): 1799-806.

7. Beitner MM, Winship I, Drummond KJ. Neurosurgical considerations in von HippelLindau disease. J Clin Neurosci 2011; 18(2): 171-80.

8. Capitanio JF, Mazza E, Motta M, Mortini P, Reni M. Mechanisms, indications and results of salvage systemic therapy for sporadic and von Hippel-Lindau related hemangioblastomas of the central nervous system. Crit Rev Oncol Hematol 2013; 86(1): 69-84. 
9. Wong WT, Liang KJ, Hammel K, Coleman HR, Chew EY. Intravitreal ranibizumab therapy for retinal capillary hemangioblastoma related to von Hippel-Lindau disease. Ophthalmology 2008; 115(11): 1957-64.

10. Omar AI. Bevacizumab for the treatment of surgically unresectable cervical cord hemangioblastoma: a case report. J Med Case Rep 2012; 6: 238. 